\title{
The Innermost Extremes of Black Hole Accretion
}

\author{
A.C. Fabian ${ }^{1, \star}$ \\ Institute of Astronomy, University of Cambridge, Madingley Road, Cambridge CB3 0HA, United Kingdom
}

Received 2015, accepted 2015

Published online later

Key words Black Holes, active galaxies, X-rays

The inner 20 gravitational radii around the black hole at the centre of luminous Active Galactic Nuclei and stellar mass Black Hole Binaries are now being routinely mapped by X-ray spectral-timing techniques. Spectral blurring and reverberation of the reflection spectrum are key tools in this work. In the most extreme AGN cases with high black hole spin, when the source appears in a low state, observations probe the region within 1 gravitational radius of the event horizon. The location, size and operation of the corona, which generates the power-law X-ray continuum, is also being revealed.

\section{Introduction}

The extremes of Black Hole accretion concentrate on the innermost regions of the accretion flow where the bulk of the luminosity is released. Here I briefly review the basic picture of this region that has emerged from X-ray observations of luminous accreting black holes.

The accretion flow is dominated by an accretion disc (Fig. 1) which for luminous objects is optically thick and physically thin, provided they are below the Eddington limit. Quasi-thermal blackbody emission from the disc accounts for much of the UV emission in Active Galactic Nuclei (AGN) and the softer X-ray emission from stellar-mass Black Hole Binaries (BHB). A variable power-law continuum is also always seen in AGN and in most spectral states of BHB. This is attributed to a spatially compact region called the corona, named after the Solar Corona, although the coronae in BHB and AGN are billions to many trillions of times more luminous. The corona is presumably powered by magnetic fields extending from the disc (Galeev, Rosner \& Vaiana 1979). Spectral-timing studies discussed below indicate that, in many well-studied luminous objects, the accretion disc extends down to the innermost stable circular orbit (ISCO) and the corona lies above its central region (Reynolds 2014).

Irradiation of the accretion disc by the power-law X-ray continuum leads to fluorescent and backscattered emission from the disc which produces another spectral component, known as the reflection spectrum (Fabian \& Ross 2010; Garcia et al 2011,13). This is rich in emission lines, particularly Fe K, which appear blurred and broadened by Doppler shifts and gravitational redshift (Fabian et al 1989; Laor 1991; Fig. 2). Measurements of the blurring extent of the reflection spectrum enable the geometry, particularly radii,

\footnotetext{
^ Corresponding author: e-mail: acf@ast.cam.aac.uk
}

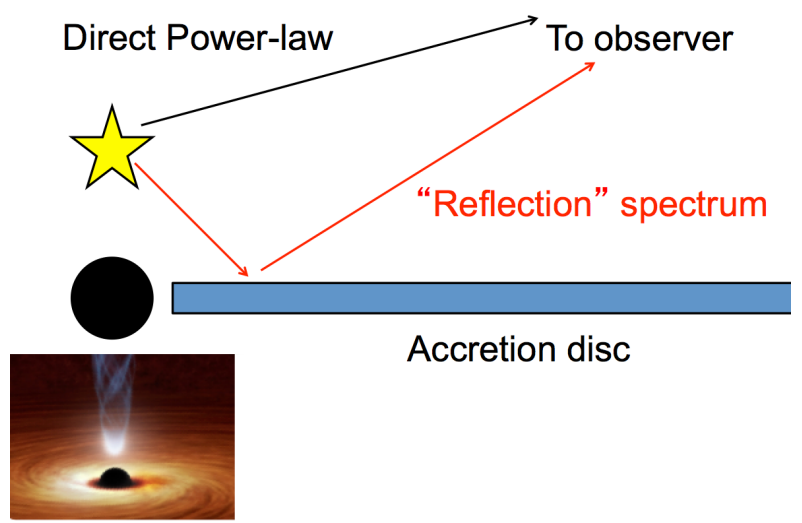

Fig. 1 Schematic representation of a thin accretion disc orbiting a black hole with the corona located on the rotation axis. The coronal power-law continuum irradiates the accretion disc producing the reflection spectrum. The extra light path in the reflection component produces a lag between variations in the direct power-law and reflection. The inset shows an artistic representation of the setup showing a possible collimated outflow or jet.

and the flow pattern of the disc and corona to be mapped in gravitational units (e.g. gravitational radii, $r_{\mathrm{g}}=G M / c^{2}$ ).

Changes in the power, location or particle outflow in the corona lead to delayed variations or reverberation in the reflection spectrum (Fabian et al 1989; Stella 1990; Reynolds et al 1999). The lag time is just the extra light crossing time from the corona to disc and out. Measurement of time lags turns the length scales, particularly the coronal height, into physical units (Uttley et al 2014).

The region that is being mapped often lies within the innermost $20 r_{\mathrm{g}}$ around the black hole and sometimes the innermost $2 r_{\mathrm{g}}$ when the black hole is spinning rapidly and the coronal height is small. Note that $50 \%$ of the accretion 


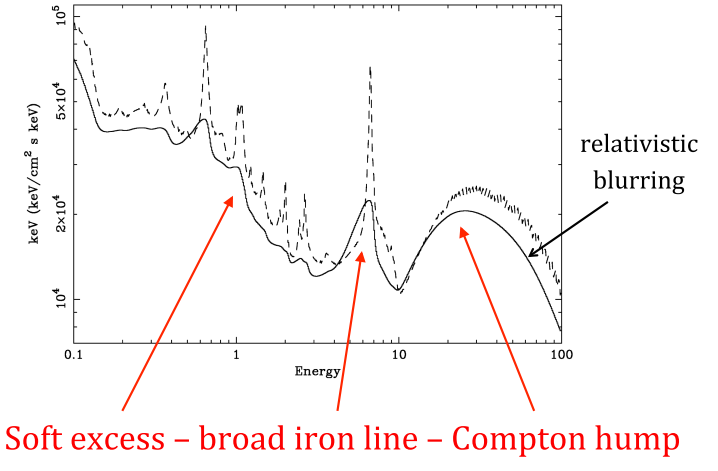

Fig. 2 The reflection spectrum showing the intrinsic emission (dashed) and the relativistically blurred emission (solid line).

power from a rapidly spinning black hole emerges from the inner $5 r_{\mathrm{g}}$ (Thorne 1994). X-ray observations of luminous, rapidly spinning, black hole sources probe closest to the event horizon. The effects of strong gravity, such as strong gravitational redshift, strong light bending, strong Shapiro effect and dragging of inertial frames, are all involved in modelling the spectral-timing results.

\section{The Reflection Spectrum}

The reflection spectrum typically consists of 3 parts: a soft excess, broad iron line and Compton hump. It is produced by the competition between electron scattering and photoelectric absorption of the incident photon. Above about 20 $\mathrm{keV}$, Compton scattering dominates creating the Compton hump, and below that energy photoelectric absorption dominates so the spectra drops away (Lightman \& White 1988). When the surface is ionized, electron scattering is again important together with re-emission through emission lines, causing the soft excess which rises into the FUV to conserve energy (Ross \& Fabian 1993, 2005; Garcia et al 2011, 2013; Fig. 2). The intrinsic reflection spectrum, which would be observed by an observer corotating with part of the disk, has many emission lines below $7 \mathrm{keV}$. Relativistic blurring redshifts and broadens the spectrum (Fabian et al 1989) so that it appears to be much smoother, particularly if the black hole spin is high, when blurring is most pronounced (Laor et al 1991; Dauser et al 2013).

The whole reflection spectrum is now commonly seen in many observations of AGN and BHB (e.g. Risaliti et al 2013; Marinucci et al 2014; Miller et al 2013a). The advent of the focussing telescope on NuSTAR (Harrison et al 2012) means that the Compton hump is now clearly seen and good models of the whole spectrum can be routinely made and used to determine parameters such as spin. Even AGN with strong variable absorption, such as NGC1365, can be modelled and shown to have an underlying reflection spectrum similar to that found in unobscured sources (Risaliti et al 2013; Walton et al 2014).

A number of AGN occasionally drop into a reflectiondominated low state 1 . Recent examples include 1 H0707495 (Fabian et al 2012; Kara et al 2015) and Mkn 335 (Parker et al 2014; Figs 5 and 6). In these cases the evidence indicates that the primary power-law is still present and irradiating the disc strongly but appears weak in our sightline due to a low coronal height which causes very strong light bending. When the corona is close to the black hole the strong gravity bends the power-law continuum away from our line of sight and down onto the disc. Most of the reflection-dominated $\mathrm{X}$-ray spectrum that we observe originates from within a radius of 3 or even just $2 r_{\mathrm{g}}$.

\section{X-ray Reverberation}

As already mentioned, there is a time lag between the detection of variations in the primary coronal power-law and variation induced in the reflection spectrum due to the greater path length taken by the photons. Such reverberation provides us with a complementary mapping device for understanding the inner regions of the accretion flow (for a review see Uttley et al 2014).

Since their discovery using XMM data of 1H0707-495 (Fabian et al 2009), high-frequency lags attributable to reverberation are now commonly seen in X-ray bright AGN which have strong rapid variability (e.g. De Marco et al 2013). Lag energy spectra reveal clear evidence that reverberation is involved through detection of the Fe $\mathrm{K}$ imprint of the reflection spectrum (Fig. 7). Low frequency lags are also seen in many sources (including BHB in which they were first detected back in the late 1980s, Miyamoto \& Kitamoto 1989) but their spectrum tends to be a rising powerlaw. They are probably due to small changes in the corona, and thus the power-law continuum.

The energy lag spectrum of both Fe K and Compton hump has now been obtained from NuSTAR, such as that shown in Fig. 7 by Kara et al (2014) using code from Zoghbi et al (2013). Since we cannot separate the direct primary continuum from the reflection spectrum, but observe both mixed together, the observed lag spectrum is diluted by the relative amount of reflection to power-law in each band. This must be taken into account when modelling the lag spectrum. The observed lag spectrum (e.g. Fig. 8) indicates that the response is seen first in the red wing of both the iron line and Compton hump and later in the less blurred parts which originate further out in the disc. In general, modelling (e.g. Cackett et al 2015; Emmanoulopoulos et al 2014) suggests that the corona is located at $3-10 r_{\text {ISCO }}$.

No alternative model for broad iron lines is able to replicate the full spectral timing results including the high frequency Fe K lags.

1 This is not the same phenomenon as the BHB low state 

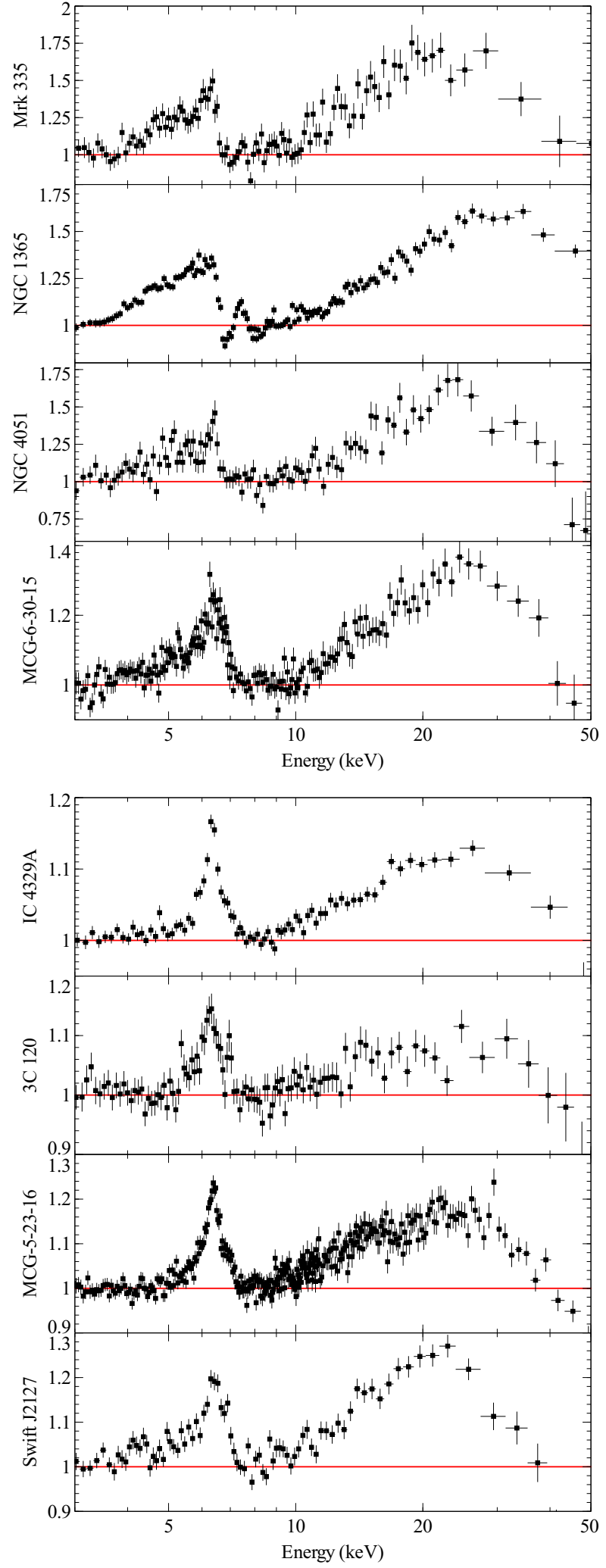

Fig. 3 NuSTAR spectral ratios to a simple power-law fitted between 3-4 keV and 8-10 keV (from Fabian et al 2014). The broad iron line and Compton hump in the reflection spectrum are evident.
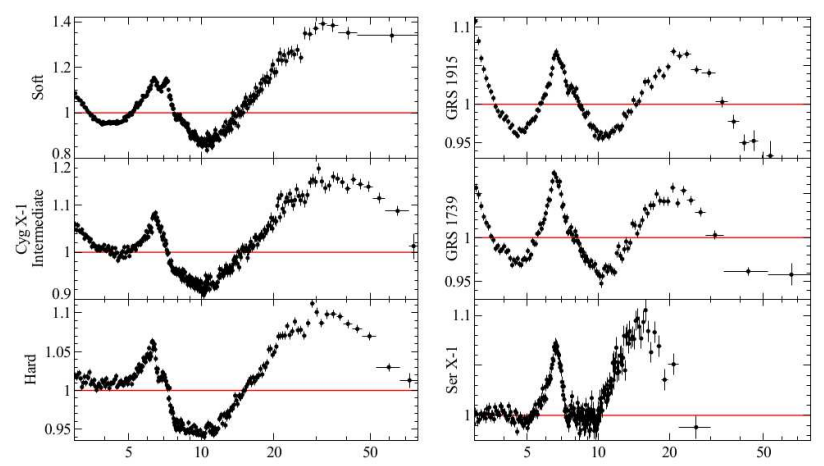

Fig. 4 NuSTAR spectral ratios for BHB (see e.g. Tomsick et al 2014; Miller et al 2013a, 2013b, 2015).

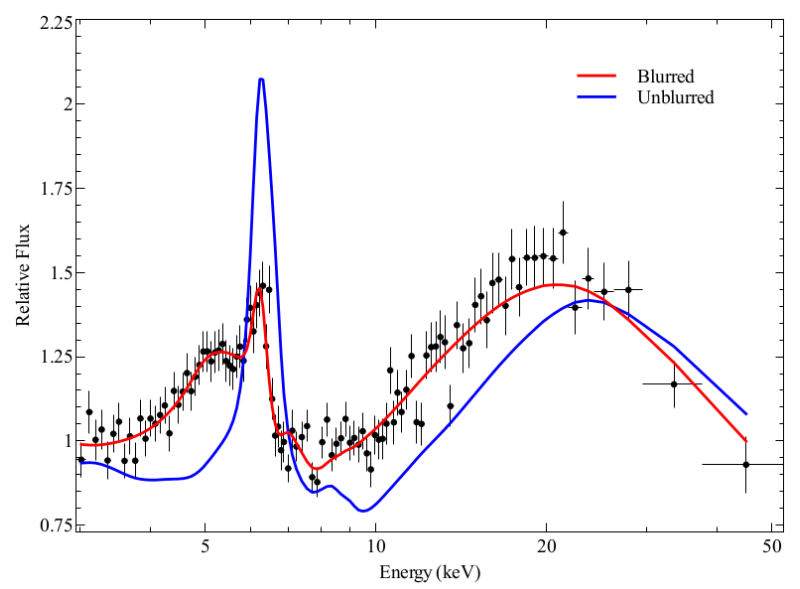

Fig. 5 Blurred and unblurred model spectra overplotted on NuSTAR data from the low state of Mkn335 (Parker et al 2014).

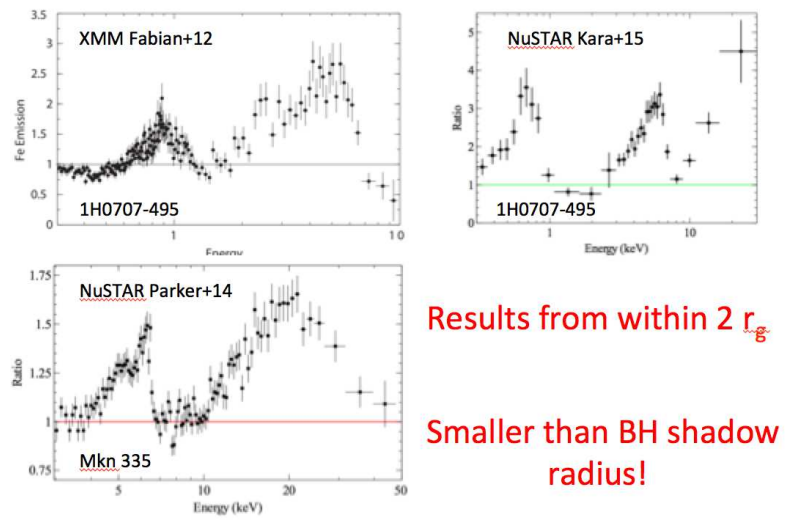

Fig. 6 Collection of AGN low state spectral ratios when the spectra are reflection dominated. Most of the emission from these spectra emerge from within $2 r_{\mathrm{g}}$. 


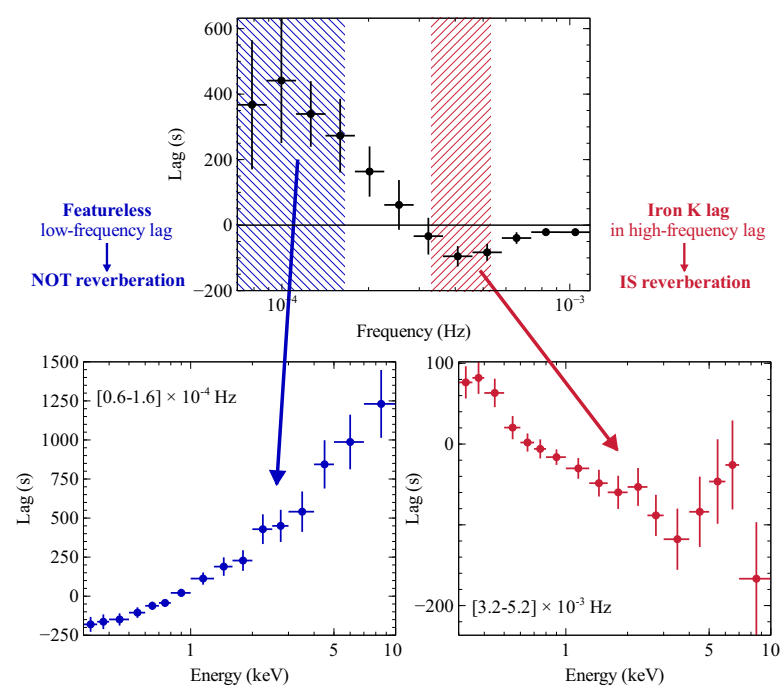

Fig. 7 Lag-frequency and lag-energy spectra of Akn 564 (Kara et al 2014). Fe K reflection features are seen in the high frequency lag-energy plot confirming that it is due to reverberation of the reflection spectrum.

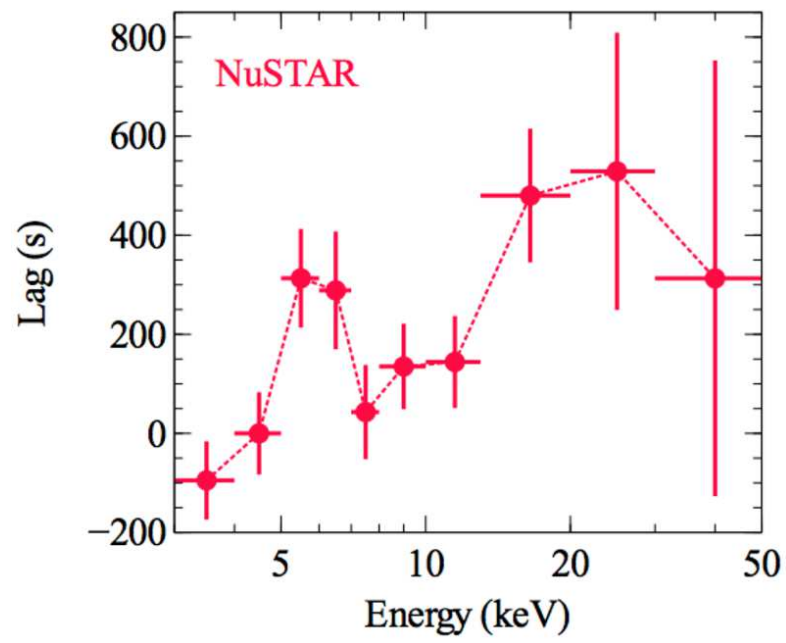

Fig. 8 Lag-energy plot of SWIFT J2127 (Kara et al 2015) showing both the broad iron line and Compton hump.

\section{Black Hole Spin}

The spin of the black hole can be determined if we identify the innermost radius required by the blurring with the ISCO. Many of the AGN sources from which the spin has been determined in this way have a spin $>0.9$, with some consistent with maximal spin of 0.998 (see talk by Chris Reynolds and Reynolds 2014). This could be considered a possible problem for the method if a wide range of spin is expected. However, there is a strong bias towards detecting objects with high spin due to the increase in radiative efficiency with spin, which can be up to a factor of 5 (Brenneman et al 2011, Vasudevan et al 2015).

To understand this bias, consider first the contribution of spinning objects to the X-ray Background. Assume that

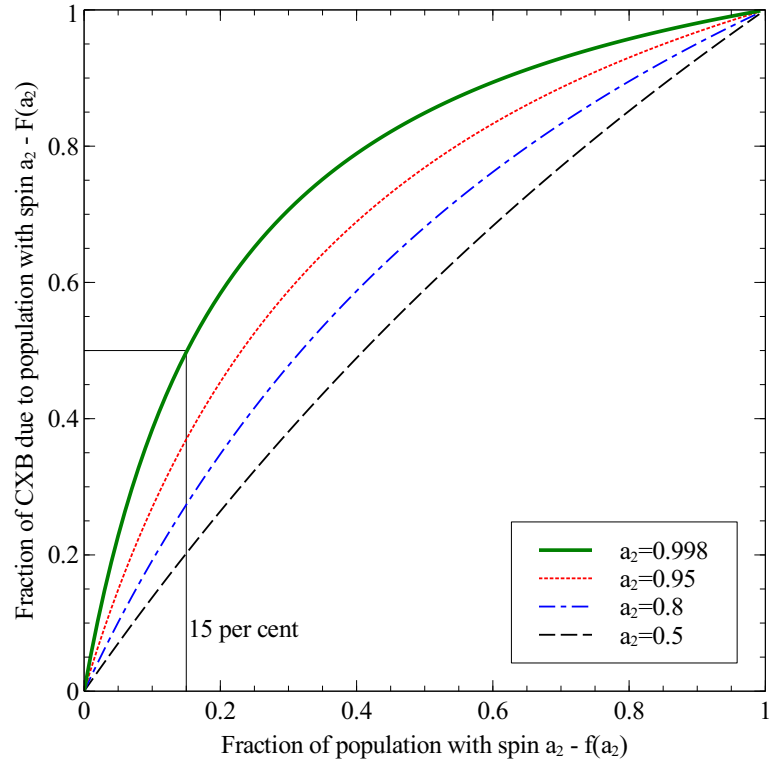

Fig. 9 Spin bias in the XRB intensity Vasudevan et al 2015). The plot assumes a two component AGN population, one fraction with spin zero the other with spin $a_{2}$. Only $15 \%$ of the population is needed with maximal spin to give half the background.

all AGN are randomly distributed between zero spin with accretion efficiency of $5.7 \%$ and near maximal spin with an efficiency about 5 times larger. Then if just a random 1/6 of the AGN have high spin then together they produce as much background intensity as the other 5/6 (Fig. 9). This means that half the background comes from $1 / 6$ of the sources. We make no assumptions here about luminosity, mass or distance, just that the distribution of high spin among sources is completely random. The $30 \mathrm{keV}$ spectral peak in the XRB may thus be due to the Compton hump in reflection from a minority population of rapidly spinning black holes.

Carrying out a similar exercise for source counts is a little more complicated and involves the $3 / 2$ power. Only 1 in 14 sources, again distributed randomly, need have high spin in order for them to produce half the bright sources counts (Fig. 10; Vasudevan et al 2015). The two component model discussed here is obviously extreme. The point is that if there is a broad intrinsic spin distribution then it should be no surprise that the brightest AGN in the Sky host some of the most rapidly spinning black holes.

\section{Coronal Physics}

Studies from several directions are revealing that corona are both physically and radiatively compact. Their physical size can be deduced from the emissivity profile of blurred reflection (typically of the broad iron line, Wilkins \& Fabian 2012), from reverberation discussed above and also from microlensing studies (e.g. Morgan et al 2012; see talk by G. Chartas). All such methods point to coronal sizes of 


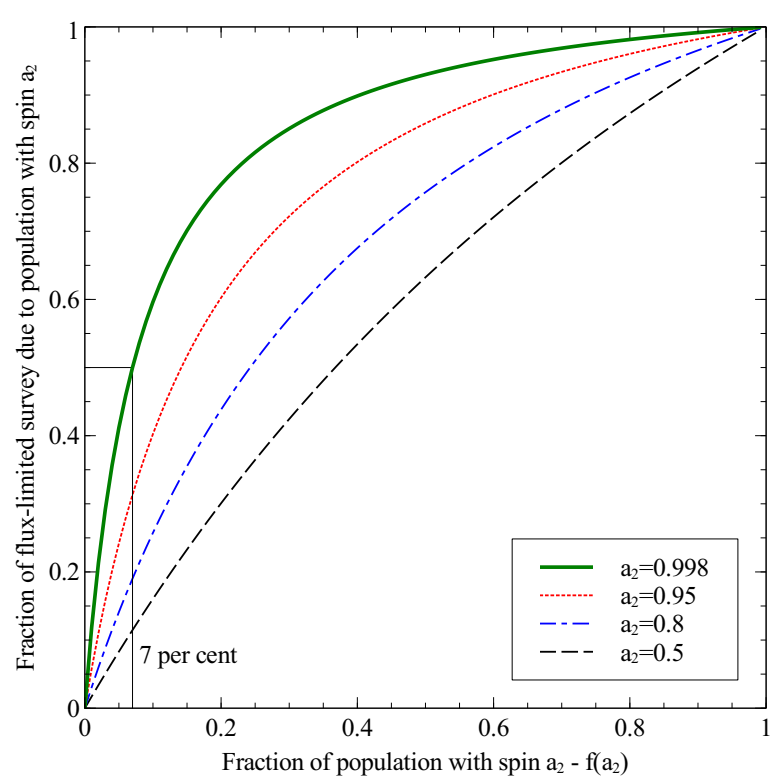

Fig. 10 Spin bias in source counts (Brenneman et al 2011; Vasudevan et al 2015). ONly 7\% ofb the population is needed with maximal spin to give half the bright sources counts.

about $10 r_{\mathrm{g}}$ or less. This means that the photon density in the corona is extremely high and thus that the probability an energetic photon trying to escape the region will collide with another photon is also high. The probability is determined by the source compactness (Guilbert et al 1983)

$$
\ell=\frac{L}{R} \frac{\sigma_{T}}{m_{\mathrm{e}} c^{3}},
$$

where $L$ and $R$ are the luminosity and size of the region, respectively. If the product of the two photon energies exceeds $2 m_{\mathrm{e}} c^{2}$ an electron-positron pair can result. The particles then help generate more photons and a runaway situation ensues which is only stabilised if the conditions change to reduce the photon energies. The net result is a maximum temperature for the region which drops lower as the compactness increases (e.g. Svensson 1984; Stern et al 1995; Fig. 11). Recent observations now indicate that $\ell \sim 10-1000$, which is about an order of magnitude larger than early indications based on source variability (Done \& Fabian 1989).

The temperature of the corona is determined from the high energy cutoff of the power-law continuum. NuSTAR has made observations of the cutoff fairly routine for bright AGN and Comptonization modelling yielding coronal temperatures $\Theta=k T / m_{\mathrm{e}} c^{2} \sim 0.03-0.8$. Interestingly an inverse correlation between $\ell$ and $\Theta$ is seen in the data, as predicted by pair production models (Fig. 12; Fabian et al 2015). It is therefore plausible that pair production controls the physics of the corona. The corona itself may be a pair plasma and the upscattered photons may originate from the magnetic field (i.e. as virtual cycltron photons).

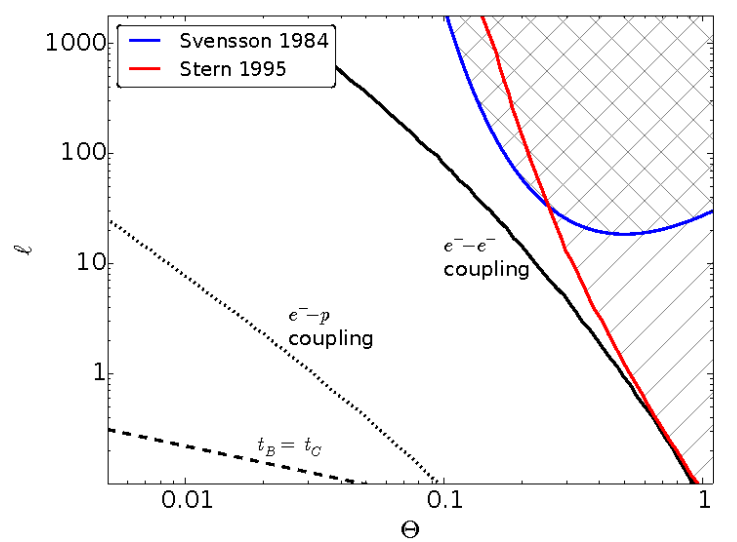

Fig. $11 \ell-\Theta$ (compactness vs temperature) diagram showing key theoretical constraints. Compton cooling is faster than a) bremsstrahlung above the dashed line, b) electron-proton thermal coulping above the dotted line and c) electron-electron thermal coupling above the solid black line. Runaway pair production occurs above the blue and red lines according to the geometry assumptions made by Svensson (1984) and Stern et al (1995). Sources are not expected to be observed in the pair runaway region.

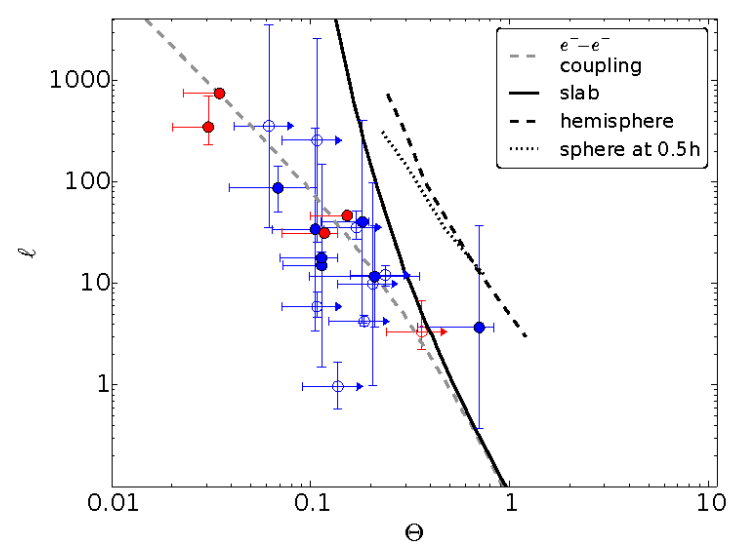

Fig. $12 \ell-\Theta$ diagram for sources observed by NuSTAR (red for BHB and blue for AGN, Fabian et al 2015). As expected, sources avoid the pair production region. When the temperature estimates are corrected for gravitational redshift the points shift closer to the pair production line. Their proximity to the line suggests that pair production plays a key role in the behaviour of the corona.

\section{Discussion}

The innermost extremes of black hole accretion can be very extreme in some currently observed AGN and BHB. Most of the X-ray luminosity originates in a powerful compact corona lying along or close to the spin axis of the black hole above (and below) the accretion disc. The corona may not be static but rotate with the disc and move vertically or expand horizontally. The particles within the corona may flow 
upward (see for example the inset in Fig. 1 and models of Beloborodov 1999 and Malzac 2001). X-ray spectral timing has enabled us to map the innermost regions of the accretion flow and corona.

Current observations are photon-limited and provide only snapshots of coronal behaviour in the X-ray brightest AGN. Understanding how the corona is powered and operates is a major step in understanding how the central engine works in the most luminous persistent objects in the Universe. More extensive long observations with XMM of the most variable X-ray bright AGN will initiate the study of coronal behaviour in a dynamical sense and demonstrate the coupling between disc and corona. Low state AGN observations lack photons which again can be compensated for with long observations. A very deep look at the low state of an AGN with a rapidly spinning black hole (e.g. Fig. 5), where most of the detected photons orginate in a reflection spectrum from within $1 r_{\mathrm{g}}$ of the horizon, will surely be worthwhile.

In the near future the broad band coverage of ASTRO$\mathrm{H}$, which is due for launch in early 2016, will further reveal the corona and reflection components of BHB and AGN. Its high resolution $\mathrm{X}$-ray microcalorimeter will enable clear separation of the effects of intervening absorbing gas and outflows. Later in the 2020s, Athena will make detailed soft $\mathrm{X}$-ray timing spectroscopy a routine exercise for many BHB and AGN.

\section{Acknowledgments}

Thanks to students, colleagues, collaborators and the NuSTAR team for much work and many discussions on the topics covered here. Michael Parker is thanked for Figs 3 and 4. The European Research Commission is thanked for Advanced Grant FEEDBACK.

\section{References}

Beloborodov, A.,: 1999, ApJ, 510, L133

Brenneman L., et al., 2011, ApJ, 736, 103

Cackett E.M., et al.: 2014, MNRAS, 438, 2980

Dauser T., et al., 2013, MNRAS, 430, 1694

De Marco B., et al.,:2013, MNRAS, 431, 2441

Done, C., Fabian, A.C.,:1989, MNRAS, 240, 81

Emmanoulopoulos, D., Papadakis, I.E., Dovciak, M., McHardy, I.M., 2014, MNRAS, 439, 3931

Fabian, A.C., Rees, M.J., Stella, L., White, N.E.,: 1989, MNRAS, 238, 729

Fabian, A.C., et. al.: 2009, Nature 459, 540

Fabian, A.C., et al.: 2012, MNRAS,419, 116

Fabian, A.C., Kara, E., Parker, M.L.,: 2014, Suzaku-MAXI2014, eds Ishida M., Petre R., Mitsuda K., p279, arXiv:1405.4150

Fabian, A.C., Lohfink, A., Kara, E., Parker, M.L., Vasudevan, R., Reynolds, C.S., 2015, MNRAS, 451, 4375

Garcia, J., Kallman, T.R., Muchotzky, R.F.,: 2011, ApJ, 731, 131

Garcia, J., et al.: 2013, ApJ, 768, 146

Guilbert, P., Fabian, A.C., Rees, M.J.,: MNRAS, 205, 593

Harrison, F., et al 2013, ApJ,770, 103
Kara, E., et al., 2013, MNRAS,434, 1129

Kara, E., et al., 2015, MNRAS, 446, 737

Kara, E., et al.,: 2015, MNRAS, 449, 234

Lightman, A.P., White, T,R.,: 1988, ApJ, 335, 57

Malzac, J., Beloborodov, A., Poutanen, J.,: 2001, MNRAS, 326, 417

Marinucci, A., et al., 2014, MNRAS, 440, 2347

Miller, J.M., et al., 2013a, ApJL, 775, L45

Miller, J.M., et al., 2013b, ApJL, 779, L2

Miller, J.M., et al., 2013a, ApJL, 799, L6

Miyamoto, S., Kitamoto, S.,: 1989, Nature, 342, 773

Morgan C.W., et al.: 2012, ApJ,756, 52

Parker, M.L.,et al.: 2014, MNRAS, 443, 1723

Risaliti, G., et al.: 2013, Nature, 494, 449

Ross, R.R., Fabian, A.C.,: 1993, MNRAS, 261, 74

Ross, R.R., Fabian, A.C.,: 2005, MNRAS, 358, 211

Stern, B., Poutanen, J., Svensson, R., Sikora M., Begelman, M.C.,: 1995, ApJ, 449

Svensson, R.,: 1984, MNRAS, 209, 1745

Thorne, K.,: 1974, ApJ, 191, 507

Tomsick, J., et al.,: 2014, ApJ, 780, 78

Uttley, P., Cackett, E.M., Fabian, A.C., Kara, E., Wilkins, D.R.,: 2014, A\&ARv, 22, 72

Vasudevan, R., Fabian, A.C., Reynolds, C.S., Dauser, T., Gallo, L.,: 2015, arXiv:1506.01027

Walton, D.J., et al.,: 2014, ApJ, 788, 76

Wilkins, D.R., Fabian A.C.,: MNRAS, 414, 1269

Zoghbi A., Reynolds, C., Cackett, E.M.,: 2013, ApJ, 777, 24 